\title{
The role of multidetector computed tomography versus digital subtraction angiography in triaging care and management in abdominopelvic trauma
}

\author{
James Thomas Patrick Decourcy $\underline{\text { Hallinan }}{ }^{1}$, MBChB, FRCR, Cher Heng $\underline{T a n}^{2}$, MBBS, FRCR, Uei $\underline{\text { Pua }}{ }^{2}$, MBBS, FRCR
}

INTRODUCTION This study aimed to assess the ability of contrast-enhanced computed tomography (CECT) to detect active abdominopelvic haemorrhage in patients with blunt trauma, as compared to digital subtraction angiography (DSA). METHODS In this retrospective study, patients who underwent DSA within 24 hours following CECT for blunt abdominal and/or pelvic trauma were identified. The computed tomography (CT) trauma protocol consisted of a portal venous phase scan without CT angiography; delayed phase study was performed if appropriate. All selected CECT studies were independently reviewed for the presence of active extravasation of contrast by two radiologists, who were blinded to the DSA results. Fisher's exact test was used to correlate the presence of extravasation on CT with subsequent confirmed haemorrhage on DSA.

RESULTS During the eight-year study period, 51 patients underwent CECT prior to emergent DSA for abdominal or pelvic trauma. Evidence of active extravasation of contrast on CECT was observed in 35 patients and active haemorrhage was confirmed on DSA in 31 of these patients; embolisation was performed in all 31 patients. Two patients who were negative for active extravasation of contrast on CECT but positive for active haemorrhage on DSA had extensive bilateral pelvic fractures and haematomas. The sensitivity, specificity, and positive and negative predictive values of CECT in detecting active abdominopelvic haemorrhage, as compared to DSA, were $93.9 \%, 77.8 \%, 88.6 \%$ and $87.5 \%$, respectively.

CONCLUSION When compared with DSA, dual-phase CECT without CT angiography shows high sensitivity and positive predictive value for the detection of active haemorrhage in patients with blunt abdominopelvic trauma.

Keywords: active haemorrhage, angiography, computed tomography, extravasation of contrast, trauma

\section{INTRODUCTION}

Computed tomography (CT) plays a key role in the diagnosis of acute abdominal and pelvic haemorrhage following blunt trauma. (1) Multidetector CT allows for rapid acquisitions, multiplanar reconstructions and complex multiphasic acquisitions, including CT angiography. ${ }^{(2,3)} \mathrm{A}$ rapid and accurate depiction of the site of haemorrhage allows accurate therapeutic decisions to be made. ${ }^{(4)}$ In the appropriate clinical setting, a negative CT could reduce unnecessary invasive procedures, as it opens up the possibility of conservative nonoperative management. ${ }^{(5)}$ In turn, detection of active haemorrhage allows for consideration of digital subtraction angiography (DSA; with or without embolisation) and/or operative management. ${ }^{(6,7)}$

Several recent studies have focused on the use of multiphasic acquisitions, in addition to the routine portal venous (PV) phase, for more accurate diagnosis; these include CT angiography (arterial phase) and delayed phase imaging. ${ }^{(8,3)}$ The clinical impact of these additional phases has yet to be determined and in a typical young population of trauma patients, the additional radiation dose involved is a potential concern. Thus, the present study aimed to retrospectively evaluate the ability of PV phase contrast-enhanced CT (CECT) with delayed phase, if performed, as compared to digital DSA (i.e. the gold standard), in detecting active abdominopelvic haemorrhage in patients with blunt trauma.

\section{METHODS}

The present study complied with the Health Insurance Portability and Accountability Act and was approved by the institutional review board of Tan Tock Seng Hospital, Singapore. The need for informed patient consent was waived as this study was retrospective.

Between April 2004 and April 2012 (i.e. an eight-year period), 101 consecutive patients underwent emergent DSA for blunt abdominopelvic trauma. The inclusion criteria for the study were: (a) underwent DSA within 24 hours following admission; and (b) underwent CECT prior to DSA. Patients who did not undergo CECT prior to DSA $(n=50)$ were excluded. A total of 51 patients (34 men and 17 women) were finally included; their mean age was 42 (range 15-83) years. The mean age of the men was 39 (range 15-79) years, while the mean age of the women was 42 (range 18-83) years. The mechanisms of blunt trauma among the 51 patients were: (a) motor vehicle collision $(n=37)$; (b) fall from a height $(n=11)$; and (c) pedestrian hit by a motor vehicle $(n=3)$.

The decision to perform DSA, with or without embolisation, was made by the trauma team (comprising an emergency physician, surgeon and radiologist) in the emergency department based on the results of the initial clinical assessment, which included haemodynamic status and CECT examination. CECT scans of the abdomen and pelvis were performed using either a 16-detector or 64-detector row scanner (SOMATOM Sensation;

${ }^{1}$ Department of Diagnostic Radiology, National University Health System, ${ }^{2}$ Department of Diagnostic Radiology, Tan Tock Seng Hospital, Singapore

Correspondence: Dr James Thomas Patrick Decourcy Hallinan, Associate Consultant, Department of Diagnostic Radiology, National University Health System, 5 Lower Kent Ridge Road, Singapore 119074. jim. hallinan@gmail.com 

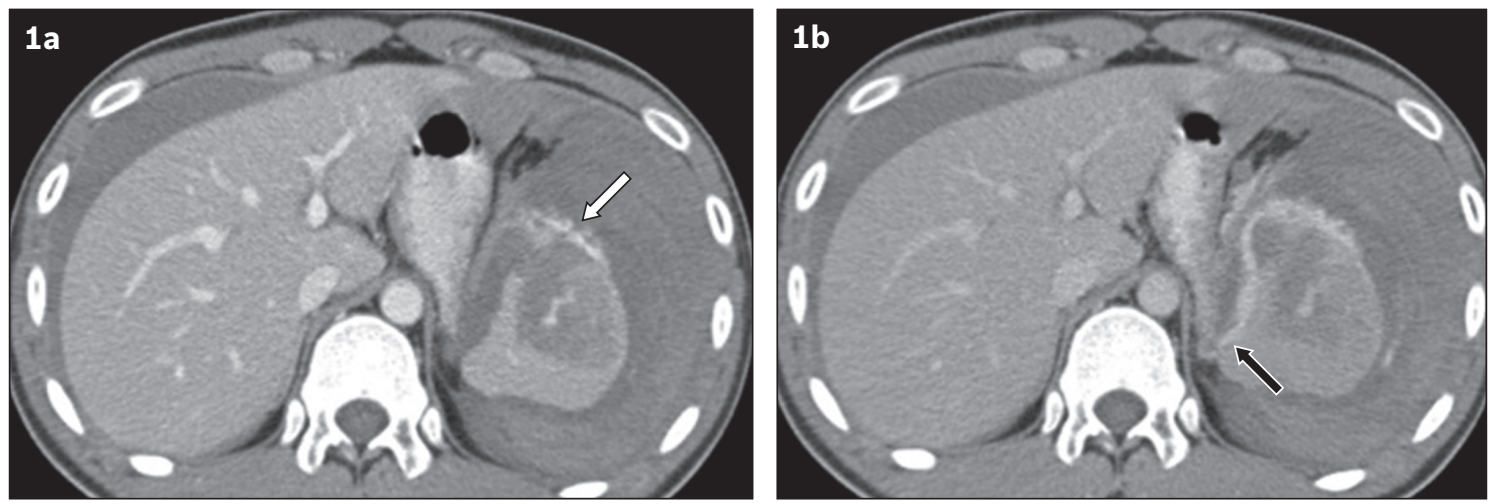

Fig. 1 A 22-year-old man after a motorcycle accident. (a) Transverse portal venous phase CT image shows active extravasation of contrast within a splenic laceration (white arrow). (b) Corresponding delayed phase CT image shows an enlarging focus of active haemorrhage extending medially (black arrow). Conventional angiography confirmed active haemorrhage in the splenic artery and successful coil embolisation of the splenic artery was performed.

Siemens Healthcare, Erlangen, Germany). The highest part of the diaphragm to the greater trochanters was included in the PV phase, using a section thickness of $0.625 \mathrm{~mm}$ and a reconstructed slice thickness of $3 \mathrm{~mm}$. All patients received a single intravenous bolus of non-ionic iodinated contrast $100 \mathrm{~mL}$ (Omnipaque 350; Nycomed Imaging AS, Oslo, Norway), which was injected at a rate of $3 \mathrm{~mL} / \mathrm{s}$ using a dual-syringe power injector. Scanning delays from the time of injection were 70 seconds for the PV phase abdominopelvic CT and five minutes for the delayed phase CT. The decision to perform delayed phase imaging of the abdomen and/or pelvis was made by the attending radiologist, based on the severity of the injuries detected on the PV phase images; if injuries such as visceral lacerations, pelvic fractures and abdominopelvic haematomas were detected, delayed phase imaging would be performed. The parameters for delayed phase imaging were identical to those used for PV phase scanning. CT angiography was not performed and oral contrast was not administered.

Two radiologists (a fourth-year resident and a consultant radiologist with five years of subspecialisation in body imaging) retrospectively reviewed all of the anonymised CT images at a picture archiving and communication system workstation. The two radiologists conducted their reviews independently. The axial, sagittal and coronal volume-rendered reformats, for both PV and delayed phase, were made available to the radiologists. The radiologists first reviewed all the available multiplanar PV phase images, but were blinded to the delayed phase images and DSA results. Thereafter, they reviewed the PV phase and delayed phase images, while remaining blinded to the DSA results.

The images were evaluated for evidence of active extravasation of contrast, which was defined as extravascular high-attenuating regions with attenuation similar to, or greater than, that of the aorta. In addition, areas of active haemorrhage were expected to increase in size and remain of higher attenuation than the aorta on the delayed phase images. ${ }^{(6)}$ Additional assessment for the presence of pseudoaneurysms was performed when the delayed phase images were made available to the radiologists. Pseudoaneurysms were identified as attenuations similar to the arterial structures that showed subsequent washout on the delayed phase. ${ }^{(6)}$

An independent review of the DSA images was performed for all 51 patients by an interventionalist who had five years of subspecialisation in vascular interventional radiology. The interventionalist was blinded to the preceding CT results during the review. The images were evaluated for evidence of vascular injury, including active haemorrhage. The images and reports were also reviewed to assess the need for embolisation. All data analysis was performed using Stata version 10.0 (Stata Corp, College Station, TX, USA). The sensitivity, specificity, positive predictive value (PPV), negative predictive value (NPV) and interobserver variability were calculated. Fisher's exact test was used to correlate the presence of extravasation on CT with subsequent confirmed haemorrhage on DSA.

\section{RESULTS}

Among the 51 patients in the study cohort, 35 (68.6\%) had evidence of active extravasation of contrast on CECT; the remaining 16 (31.4\%) patients did not. PV phase study alone was conducted for 15 patients, while delayed phase study in addition to PV phase study was conducted for 36 patients (6 abdominal, 8 pelvic, and 22 abdominal and pelvic).

The interobserver agreement for the detection of active extravasation of contrast was excellent (Cohen's kappa value $0.95, \mathrm{p}<0.001)$. The radiologists disagreed on a single case; the subsequent consensus review determined active extravasation from the right hepatic lobe and active haemorrhage from the right hepatic artery was confirmed on DSA. The PV and delayed phase images in this case were degraded by beam-hardening artefacts from the right forearm.

Active haemorrhage was confirmed on DSA in 31 (88.6\%) of the 35 patients, with subsequent embolisation performed in all 31 patients. The specific sites of haemorrhage were the liver $(\mathrm{n}=8)$, spleen ( $\mathrm{n}=3$; Fig. 1 ) and pelvis ( $\mathrm{n}=20$; Fig. 2 ). Among these 31 patients, 17 (54.8\%) underwent Gelfoam $₫$ embolisation, while $14(45.2 \%)$ underwent coil embolisation with or without Gelfoam. Technical success was achieved in all 17 patients who underwent Gelfoam embolisation, but only in 13 (92.9\%) patients who underwent coil embolisation with or without Gelfoam. The patient in whom coil embolisation did not achieve technical success required further operative management due to continuous haemorrhage of the right hepatic artery. 

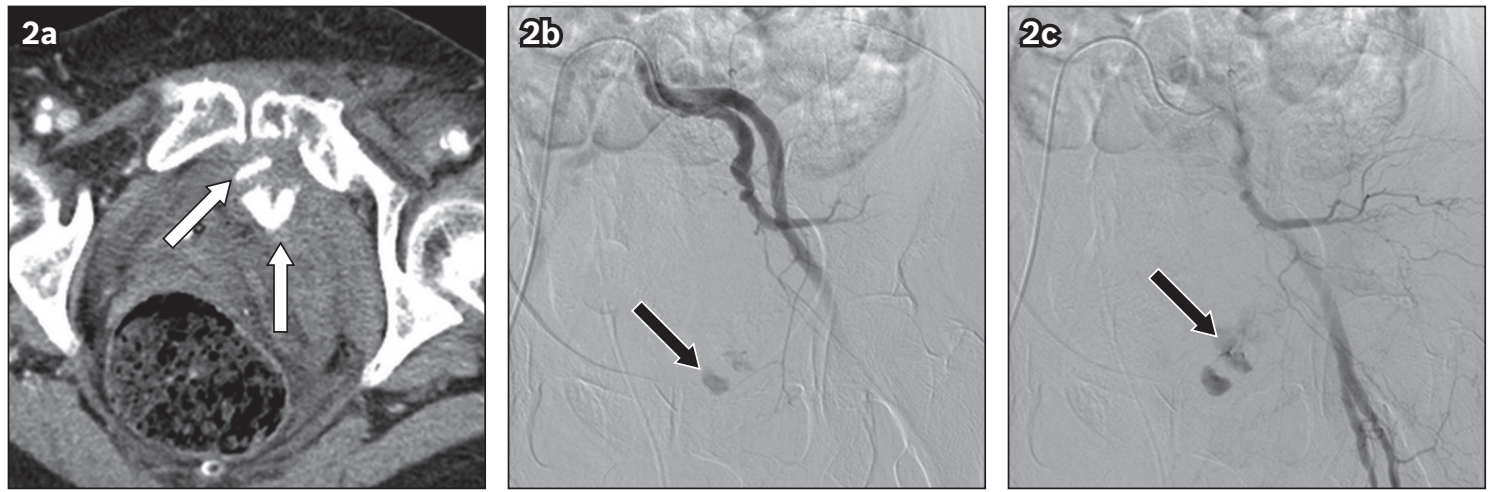

Fig. 2 An 83-year-old woman who fell from a height. (a) Transverse portal venous phase CT image shows a large haematoma and active extravasation of contrast (white arrows) in the pelvis; (b \& c) conventional angiograms show the presence of active haemorrhage in the left internal pudendal artery (black arrows), which increases in extent in (c). Successful Gelfoam embolisation was performed.

In the $4(11.4 \%)$ patients for whom subsequent DSA was negative for active haemorrhage, the sites of active extravasation on CECT were the liver (extensive lacerations observed, $\mathrm{n}=2$ ) and the pelvis (several displaced pelvic fractures and surrounding haematomas were apparent, $\mathrm{n}=2$ ). Prophylactic Gelfoam embolisation was performed in all four patients based on the CECT findings and clinical assessment, which included haemodynamic status.

Among the 16 patients without evidence of extravasation on CECT, active haemorrhage on DSA was observed in $2(12.5 \%)$ patients. Additional imaging (i.e. delayed phase $\mathrm{CT}$ ) of the pelvis was performed in one of these patients. Both patients had extensive pelvic fractures with surrounding haematomas and underwent successful Gelfoam embolisation. Evidence of pseudoaneurysms was detected in another 2 (12.5\%) of the 16 patients; one patient had a pseudoaneurysm arising from the splenic artery, while the other had a pseudoaneurysm arising from the right hepatic artery (Fig. 3). The pseudoaneurysms were confirmed on DSA and coil embolisation was performed successfully for both patients.

Active haemorrhage on DSA was not detected in the remaining $12(75.0 \%)$ patients without evidence of extravasation on CECT. One patient with multiple lacerations of the spleen remained persistently hypotensive and underwent coil embolisation of the splenic artery; seven patients with pelvic fractures/haematomas and two patients with hepatic lacerations underwent prophylactic Gelfoam embolisation.

Detection of active abdominopelvic haemorrhage using PV phase and dual-phase CECT, as compared to DSA, had a sensitivity, specificity, PPV and NPV of 93.9\% (95\% confidence interval [CI] $80.4 \%-98.3 \%), 77.8 \%$ (95\% Cl 54.8\%-91.0\%), 88.6\% (95\% Cl $74.0 \%-95.5 \%)$ and $87.5 \%$ (95\% Cl 64.0\%-96.5\%), respectively (Table I). Using Fisher's exact test, we found that the association between the presence of active extravasation of contrast on dualphase CECT and the subsequent presence of active haemorrhage on DSA was statistically significant $(p=0.0001)$.

Among the 51 patients, 36 underwent both PV phase and delayed phase imaging. The use of PV phase images alone had a sensitivity, specificity, PPV and NPV of $86.4 \%(95 \%$ $\mathrm{Cl} 66.7 \%-95.3 \%), 71.4 \%(95 \% \mathrm{Cl} 45.4 \%-88.3 \%), 82.6 \%$
Table I. Active extravasation of contrast on portal venous phase and dual-phase contrast-enhanced computed tomography (CECT) versus active haemorrhage on digital subtraction angiography (DSA).

\begin{tabular}{lccc}
\hline \multirow{2}{*}{$\begin{array}{l}\text { Active extravasation on } \\
\text { CECT (No.) }\end{array}$} & \multicolumn{3}{c}{ Active haemorrhage on DSA (No.) } \\
\cline { 2 - 4 } & Positive & Negative & Total \\
\hline Positive & 31 & 4 & 35 \\
Negative & 2 & 14 & 16 \\
Total & 33 & 18 & 51 \\
\hline
\end{tabular}

Table II. Active extravasation of contrast on dual-phase contrast-enhanced computed tomography (CECT), using only portal venous phase imaging, versus active haemorrhage on digital subtraction angiography (DSA).

\begin{tabular}{lccc}
\hline \multirow{2}{*}{$\begin{array}{l}\text { Active extravasation on } \\
\text { CECT (No.) }\end{array}$} & \multicolumn{3}{c}{ Active haemorrhage on DSA (No.) } \\
\cline { 2 - 4 } & Positive & Negative & Total \\
\hline Positive & 19 & 4 & 23 \\
Negative & 3 & 10 & 13 \\
Total & 22 & 14 & 36 \\
\hline
\end{tabular}

Table III. Active extravasation of contrast on dual-phase contrast-enhanced computed tomography (CECT), using both portal venous and delayed phase imaging, versus active haemorrhage on digital subtraction angiography (DSA).

\begin{tabular}{lccc}
\hline \multirow{2}{*}{$\begin{array}{l}\text { CECTive extravasation on } \\
\text { (No.) }\end{array}$} & \multicolumn{3}{c}{ Active haemorrhage on DSA (No.) } \\
\cline { 2 - 4 } & Positive & Negative & Total \\
\hline Positive & 21 & 4 & 25 \\
Negative & 1 & 10 & 11 \\
Total & 22 & 14 & 36 \\
\hline
\end{tabular}

(95\% $\mathrm{Cl} 62.9 \%-93.0 \%)$ and $76.9 \%(95 \% \mathrm{Cl} 49.7 \%-91.8 \%)$, respectively, while the use of both PV and delayed phase images had a sensitivity, specificity, PPV and NPV of $95.5 \%(95 \% \mathrm{Cl}$ $78.2 \%-99.2 \%), 71.4 \%$ (95\% Cl 45.4\%-88.3\%), 84.0\% (95\% Cl $65.3 \%-93.6 \%)$ and $90.9 \%(95 \% \mathrm{Cl} 62.3 \%-98.4 \%)$, respectively (Tables II \& III). Although the differences between the use of PV phase images alone and the use of both PV and delayed phase images were not statistically significant $(p>0.05)$, the use of delayed phase images helped to detect active extravasation from the right hepatic lobe and the left pelvis in two cases. These sites of haemorrhage were confirmed on subsequent DSA. 

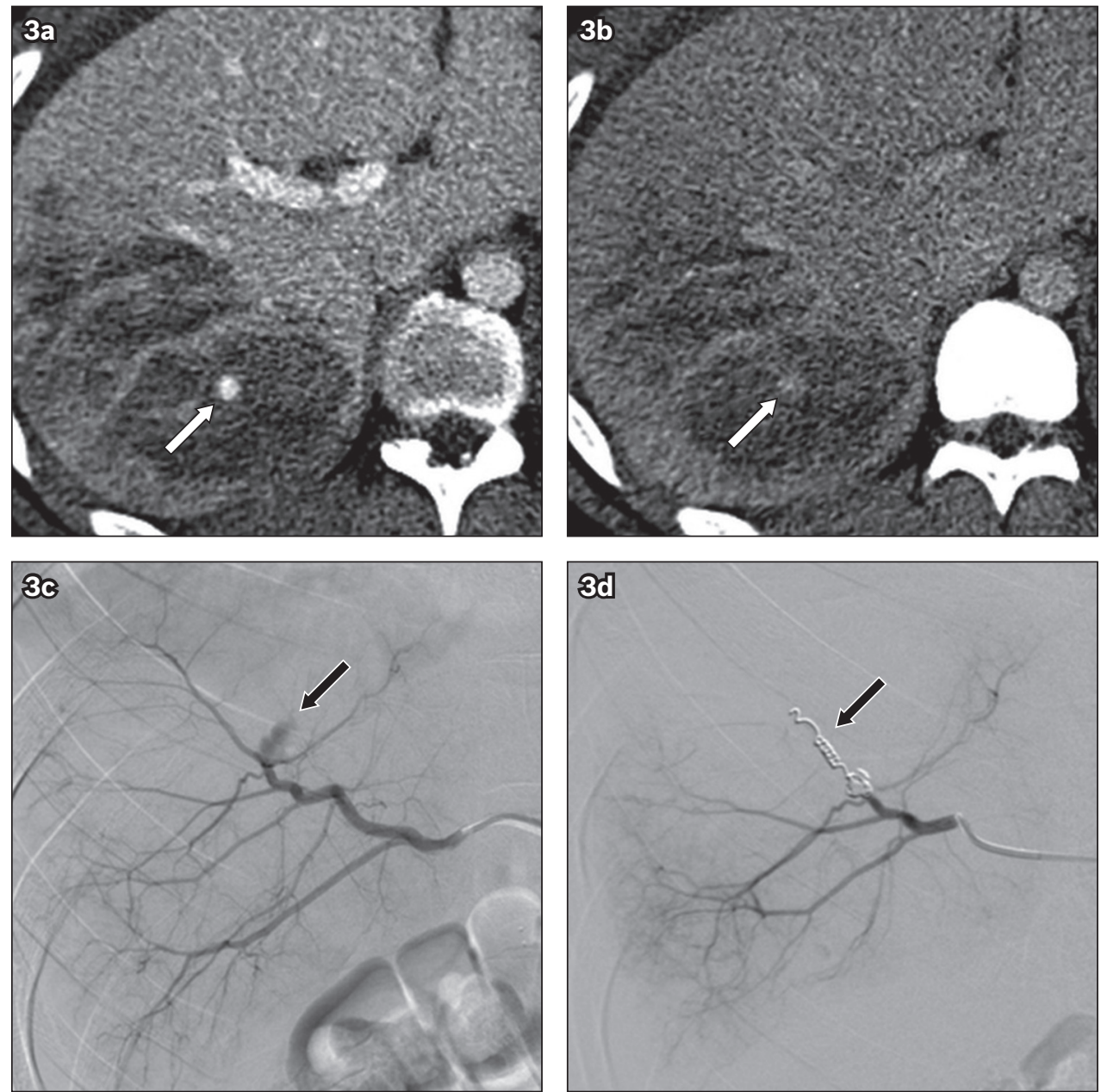

Fig. 3 An 18-year-old man who was involved in a motorcycle accident. (a) Transverse portal venous phase CT image shows a suspected focus of active extravasation (white arrow) within a right hepatic lobe laceration. (b) Corresponding delayed phase CT image shows no enlargement of the focus (white arrow) with washout, consistent with a pseudoaneurysm. (c) Conventional angiogram confirms a focal, well-circumscribed, lobulated pseudoaneurysm in the right hepatic artery (black arrow). No active haemorrhage was detected. (d) Angiogram shows successful coil embolisation (black arrow) of the pseudoaneurysm and the associated branch of the right hepatic artery.

\section{DISCUSSION}

CT is a crucial diagnostic tool for the detection of active abdominopelvic haemorrhage in trauma patients. A recent large retrospective study conducted by Huber-Wagner et al in 2013 showed that the use of $\mathrm{CT}$ during trauma resuscitation in 16,719 patients significantly increased the survival rate of both haemodynamically stable and unstable major trauma patients. ${ }^{(9)}$ Initial CT studies in the 1990s used single detector systems with long acquisition times; these systems were typically limited to single PV phase studies. ${ }^{(6)}$ With the introduction of multidetector $\mathrm{CT}$, there was increased scope for rapid multiphasic acquisitions, including arterial, PV and delayed phase studies.

PV phase studies are useful for detecting active extravasation, which is the most reliable indicator of active bleeding on subsequent DSA. (1) Thus, PV phase studies can be used to triage patients for management; patients with active extravasation should be considered for invasive procedures such as DSA or surgery, whereas patients who are negative for active extravasation or extensive haematomas may be managed conservatively.

Single PV phase studies have been reported to have limited sensitivity and specificity in detecting active extravasation or haemorrhage. ${ }^{(10,11)}$ The additional use of an arterial phase study can improve the sensitivity for detecting active haemorrhage. Abdominal and pelvic arteries are optimally opacified on CT angiography, allowing detection of the site of haemorrhage and characterisation of arterial versus venous haemorrhage. In addition, the rate of haemorrhage and injury severity can be qualitatively assessed by comparing the images obtained on the arterial, PV and delayed phases. Post-processing techniques, such as maximum intensity projections or three-dimensional reconstructions, can be performed using the arterial phase; this allows for the fine delineation of arterial anatomy, which is useful in treatment planning. ${ }^{(3)}$

Delayed phase studies performed in addition to arterial and PV phase studies may improve visualisation of active extravasation, provide qualitative assessment of the rate of haemorrhage and allow characterisation of arterial injuries (e.g. differentiating pseudoaneuryms from extravasation). Urinary extravasation may also be detected using delayed phase imaging. In trauma protocols, use of the plain, unenhanced phase is not routine. One potential benefit is that the plain, unenhanced phase enables the differentiation of bony fracture fragments from extravasation; this, however, should not be an issue when several phases are 
utilised and the extravasation is seen to fade into an enlarged, enhancing haematoma. ${ }^{(12)}$

Currently, no standard CT trauma protocol exists. There is a trend toward triphasic studies, which utilise an arterial phase, as reflected in the guidelines issued by the Royal College of Radiologists in the United Kingdom. ${ }^{(13)}$ The guidelines recommend that haemodynamically unstable patients with high risk of vascular injury undergo arterial, PV and delayed phase triphasic $\mathrm{CT}$. As previously mentioned, this is aimed at increasing the sensitivity for detecting life-threatening haemorrhage (e.g. arterial versus venous haemorrhage) and to facilitate better treatment planning. ${ }^{(13)}$

In the present study, we utilised dual-phase CECT without $\mathrm{CT}$ angiography and demonstrated that it had high sensitivity and PPV for the detection of active haemorrhage in patients with blunt abdominopelvic trauma when directly compared with DSA. The sensitivity of the dual-phase CECT without CT angiography in the present study (i.e. $93.9 \%$ ) was similar to that of a study conducted by Maturen et al in 2007, which reported a sensitivity of $94.1 \%$ in the detection of active haemorrhage in the chest, abdomen and pelvis using PV phase CECT in 48 patients (when compared to DSA performed within 24 hours). ${ }^{(14)}$ In 2004, Ryan et al examined the use of CECT in diagnosing active arterial extravasation in 498 patients with blunt abdominopelvic trauma. ${ }^{(15)}$ In that study, the authors identified 28 patients who had active extravasation of contrast, with the majority of active arterial haemorrhage originating from a pelvic source. Only nine of the 28 patients became sufficiently haemodynamically unstable to warrant angiography and seven of the nine patients demonstrated active bleeding requiring embolisation.

In 2008, Anderson et al conducted a study to review the use of pelvic CT angiography with 64-channel multidetector row $\mathrm{CT} .{ }^{(8)}$ The authors integrated pelvic CT angiography into the standard thoracoabdominal CT examination for blunt trauma. ${ }^{(6,8,16)}$ Pelvic CT angiography was performed 23 seconds after contrast injection at a rate of $5 \mathrm{~mL} / \mathrm{s} .{ }^{(8)} \mathrm{CT}$ of the chest (optional, 30 seconds), abdomen and pelvis (70 seconds), as well as delayed images of the abdomen and pelvis (five minutes) were acquired. The authors also used dose reduction techniques when obtaining the delayed phase images.(8) Pelvic CT angiography was performed in 53 patients for whom PV and delayed phase images were available; in 21 patients, vascular injury with arterial extravasation, arterial occlusion and venous haemorrhage was detected. 11 of these 21 patients underwent further angiography, with embolisation performed in seven of the 11 patients. It was noted that larger foci of active arterial haemorrhage, with serial enlargement on the PV and delayed phases, were associated with an increased rate of angiography. Importantly, all 32 patients who did not show vascular injury on CT were successfully treated conservatively. ${ }^{(8)}$

Another study by Pinto et al in 2010 examined the role of triphasic CECT (arterial, PV and delayed phases) of the whole body (craniocerebral to pelvic) versus DSA in the assessment of arterial haemorrhage in patients with pelvic vascular trauma. ${ }^{(17)}$ Multidetector CT identified pelvic bleeding in 21 (75.0\%) of the 28 patients; most of the cases were detected in the delayed phase. Active arterial extravasation was detected in 12 of the 21 patients and haemorrhage was confirmed on DSA in ten of the 12 patients. CECT had a sensitivity and PPV of $42.9 \%$ and $100 \%$, respectively. ${ }^{(17)}$

In the present study, CT angiography was not indicated in our trauma protocol, as the literature did not support the routine use of arterial phase studies in the abdomen and pelvis. In a study by Maturen et al, ${ }^{(14)}$ the authors used PV phase trauma CT alone and had high sensitivity for the detection of active haemorrhage in the chest, abdomen and pelvis. Although recent studies by Anderson et $\mathrm{al}^{(8)}$ and Pinto et $\mathrm{l}^{\left({ }^{(17)}\right.}$ provide evidence on the role of $\mathrm{CT}$ angiography in pelvic trauma, no clear role for $\mathrm{CT}$ angiography in abdominal trauma has been shown in the literature. ${ }^{(3)}$

The dual-phase CECT protocol used in the present study is useful in guiding clinical decisions for patients with blunt pelvic trauma, as it is able to provide information on the presence and site of the haemorrhage. Anderson et al showed that pelvic CT angiography could enable the differentiation of arterial from venous haemorrhage. ${ }^{(8)}$ In other words, the use of pelvic CT angiography may be able to increase the rate of conservative management and reduce the number of unnecessary DSA studies performed. In the present study, we had a high PPV (i.e. 88.6\%) and most of the patients were found to have an arterial source of haemorrhage, suggesting that the incidence of venous haemorrhage is low.

The use of delayed phase images in 36 of the 51 patients in the present study allowed for the additional detection of active extravasation of contrast in two cases, as compared to when only PV phase was used. Subsequent DSA confirmed active haemorrhage in these two patients in the right hepatic lobe and left pelvis, respectively. These findings indicate that the use of delayed phase images could improve the diagnostic accuracy of CECT. However, it should be noted that in the majority of dual-phase studies, active extravasation of contrast was clearly detected on the PV phase and the use of a delayed phase did not appear to provide any additional information. In the present study, the distinction between pseudoaneurysms and active extravasation was achieved in two cases via delayed phase studies. This, however, did not alter the patients' subsequent clinical management, which involved DSA confirmation and coiling.

Detection of active abdominopelvic haemorrhage using dualphase CECT had a high NPV (i.e. $87.5 \%$ ) in the present study; only two of the 16 patients without evidence of extravasation on CECT showed active haemorrhage on DSA. Both patients had pelvic haemorrhage with extensive pelvic fractures and surrounding haematomas, and Gelfoam embolisation without coils was successfully performed. One of the two patients did not undergo delayed phase imaging of the pelvis, which might have allowed the haemorrhage to be detected.

The present study was not without limitations. The retrospective nature of the investigation limited our understanding of the decision for initial CECT and subsequent DSA, especially given the plethora of clinical information that was considered. The decision to perform a delayed phase study was also dependent on 
the initial impression of the radiologist, which was not recorded, although it was likely to be based on the severity of the injuries detected (e.g. the presence/absence of visceral lacerations, pelvic fractures and abdominopelvic haematomas). Another limitation was the incomplete follow-up of patients after DSA. Follow-up would have been useful to determine whether active extravasation of contrast on CT and active haemorrhage on DSA is associated with increased morbidity and mortality. Murakami et al looked at the clinical implications of active extravasation in the abdomen and pelvis (on 64-channel multidetector CT) in 125 patients and found that patients with solid organ, mesenteric and pelvic haemorrhage were statistically more likely to need subsequent intervention or die, as compared to those with intramuscular, subcutaneous or retroperitoneal active extravasation $(p<0.006) .{ }^{(18)}$ A prospective study assessing the impact of the CECT and DSA findings on subsequent management, morbidity and mortality would be useful.

With the rapid development of multidetector $\mathrm{CT}$, there is potential for the integration of multiphasic CECT examinations using a single bolus of intravenous contrast. Currently, the clinical yield of additional abdominopelvic CT angiography and routine delayed phase imaging has not been clearly defined. Information obtained from increased imaging comes at the cost of a higher radiation dose. Based on the findings of the present study, there appears to be scope for reducing the number and coverage of additional delayed phase studies. This should be further evaluated with a prospective study that involves the documentation of the need for a delayed phase. In addition, a reduced-dose delayed phase that has been utilised in prior studies ${ }^{(8)}$ may be considered. To conclude, dual-phase abdominopelvic CECT using PV and delayed phase, if appropriate, allows for the accurate detection of active haemorrhage when compared directly with DSA.

\section{REFERENCES}

1. Shanmuganathan K, Mirvis SE, Sover ER. Value of contrast-enhanced CT in detecting active hemorrhage in patients with blunt abdominal or pelvic trauma. AJR Am J Roentgenol 1993; 161:65-9.

2. Willmann JK, Roos JE, Platz A, et al. Multidetector CT: detection of active hemorrhage in patients with blunt abdominal trauma. AJR Am J Roentgenol 2002; 179:437-44.

3. Uyeda JW, Anderson SW, Sakai O, Soto JA. CT angiography in trauma. Radiol Clin North Am 2010; 48:423-38.

4. Yao DC, Jeffrey RB Jr, Mirvis SE, et al. Using contrast-enhanced helical CT to visualize arterial extravasation after blunt abdominal trauma: incidence and organ distribution. AJR Am J Roentgenol 2002; 178:17-20.

5. van der Vlies $\mathrm{CH}$, Olthof DC, Gaakeer $\mathrm{M}$, et al. Changing patterns in diagnostic strategies and the treatment of blunt injury to solid abdominal organs. Int J Emerg Med 2011; 4:47.

6. Kertesz JL, Anderson SW, Murakami AM, et al. Detection of vascular injuries in patients with blunt pelvic trauma by using 64-channel multidetector CT. Radiographics 2009; 29:151-64.

7. Miller PR, Moore PS, Mansell E, Meredith JW, Chang MC. External fixation or arteriogram in bleeding pelvic fracture: initial therapy guided by markers of arterial hemorrhage. J Trauma 2003; 54:437-43.

8. Anderson SW, Soto JA, Lucey BC, et al. Blunt trauma: feasibility and clinical utility of pelvic CT angiography performed with 64-detector row CT. Radiology 2008; 246:410-9.

9. Huber-Wagner S, Biberthaler P, Häberle S, et al; TraumaRegister DGU. Whole-body CT in haemodynamically unstable severely injured patients--a retrospective, multicentre study. PLoS One 2013; 8:e68880.

10. Brown CV, Kasotakis G, Wilcox A, et al. Does pelvic hematoma on admission computed tomography predict active bleeding at angiography for pelvic fracture? Am Surg 2005; 71:759-62.

11. Cerva DS Jr, Mirvis SE, Shanmuganathan K, Kelly IM, Pais SO. Detection of bleeding in patients with major pelvic fractures: value of contrast-enhanced CT. AJR Am J Roentgenol 1996; 166:131-5.

12. Hallinan JT, Tan CH, Pua U. Emergency computed tomography for acute pelvic trauma: where is the bleeder? Clin Radiol 2014; 69:529-37.

13. The Royal College of Radiologists. Appendix 2. Examples of polytrauma protocols. In: Standards of practice and guidance for trauma radiology in severely injured patients [online]. Available at: http://www.rcr.ac.uk/docs/ radiology/pdf/BFCR\%2811\%293_trauma.pdf. Accessed August 1, 2015.

14. Maturen KE, Adusumilli S, Blane CE, et al. Contrast-enhanced CT accurately detects hemorrhage in torso trauma: direct comparison with angiography. J Trauma 2007; 62:740-5

15. Ryan MF, Hamilton PA, Chu P, Hanaghan J. Active extravasation of arterial contrast agent on post-traumatic abdominal computed tomography. Can Assoc Radiol J 2004; 55:160-9.

16. Uyeda J, Anderson SW, Kertesz J, Rhea JT, Soto JA. Pelvic CT angiography in blunt trauma: imaging findings and protocol considerations [corrected]. Abdom Imaging 2010; 35:280-6.

17. Pinto A, Niola R, Tortora G, et al. [Role of multidetector-row CT in assessing the source of arterial haemorrhage in patients with pelvic vascular trauma. Comparison with angiography.] Radiol Med 2010; 115:648-67. English, Italian.

18. Murakami AM, Anderson SW, Soto JA, et al. Active extravasation of the abdomen and pelvis in trauma using 64MDCT. Emerg Radiol 2009; 16:375-82. 